\title{
A Inclusão Digital de Trabalhadores mais velhos - quais são os desafios?
}

\author{
Johannes Doll ${ }^{*}$
}

\begin{abstract}
Resumo
O presente trabalho representa uma contribuição para o processo de inclusão digital, que é hoje necessário para uma participação ativa na nossa sociedade. A necessidade de ter conhecimentos sobre o mundo informatizado representa um desafio especial para os trabalhadores com mais que 40 anos, os quais não tiveram, durante sua formação escolar, acesso a conhecimentos referente à informática, mas que precisam destas informações agora para se manter em um mundo de trabalho em rápido processo de modernização. O presente trabalho analisa dados recolhidos durante os últimos quatro anos sobre trabalhadores com mais que 40 anos da indústria metalúrgia da região da Grande Porto Alegre, confrontando-os com dados atuais sobre a exclusão digital no Brasil. Os resultados da análise permitem perceber as necessidades, as possibilidades e os desafios que se colocam para uma inclusão digital efetiva desse grupo específico.
\end{abstract}

Palavras Chaves: inclusão digital, envelhecimento, trabalho

The digital inclusion of older workers - what are the challenges?

\begin{abstract}
This article representes a contribuition to the process of digital inclusion, necessary today for an active participation in our society. The necessity of having konwoledge about the world of informatics representes a special challenge for the blue-collor workers at age 40 and more. During their schooling, they hadn't acess to this kind of information, but know, they are needing it to maintain themselves employed in a fastly modernizating world of work. The article analyses data collected during the last four years about worker at age fourty and more in the steelcompanies of Grand Porto Alegre, Brazil, confronting them with actual data about digital exclusion in Brazil. The results of this analyse permite to determinate the necessity, the possibility and the challenges of an affective digital inclusion of this specific group.
\end{abstract}

Keywords: digital inclusion, aging, work

\section{Introdução}

A inclusão digital representa uma preocupação crescente, pois o domínio das novas tecnologias de computação e de comunicação tornam-se cada vez mais condição necessária para participar de forma ativa na sociedade atual. O Brasil ainda está marcado por um índice de inclusão digital baixo, já que em 2001 somente 12,46\% da população tinham acesso a um computador e 8,31\% à Internet (PNAD, 2001). Esta exclusão digital não só traduz, de certa forma, as desigualdades existentes na sociedade brasileira, mas pereniza as mesmas, já que exclui a maior parte da população dos meios considerados necessários para participar futuramente do mercado de trabalho, de processos educacionais e do acesso a informações importantes.

Para mudar este quadro são necessárias estratégias adequadas, como políticas públicas específicas, as quais precisam considerar, para que elas tenham realmente sucesso, dois aspectos fundamentais: em primeiro lugar, uma participação ativa da população em questão (Neri 2003, p. 15); em segundo lugar, um olhar diferenciado sobre os diferentes grupos de excluídos e suas necessidades e potencialidades específicas. Dentro desta perspectiva, o presente trabalho analisa a situação de um determinado grupo, a situação de trabalhadores da indústria metalúrgica com mais que 40 anos, considerados no seu contexto como trabalhadores mais velhos. A atenção a este grupo específico justifica-se pelo fato que, dentro do processo de envelhecimento populacional do Brasil, este grupo

\footnotetext{
* Professor adjunto, Doutor em Filosofia, professor do DEC e do PPGEDU/FACED/UFRGS, Vice-Diretor da FACED/UFRGS, Coordenador do Núcleo de Estudos Interdisciplinares sobre o Envelhecimento/UFRGS; e-mail: jdoll@ edu.ufrgs.br. V. $2 \mathrm{~N}^{\circ} 1$, Março, 2004
} 
apresenta as maiores taxas de crescimento no mercado de trabalho (veja figura 1). Além da importância demográfica, trata-se de um grupo que, na sua formação escolar, ainda não teve acesso ao mundo digital, mas que precisa enfrentar o processo de modernização acelerada dos meios de produção (Jornada 1999), seja no seu ambiente de trabalho, para se manter empregado, seja para não ficar restrito às poucas áreas, onde a modernização ainda não chegou. Finalmente, trata-se de um grupo que, por sua idade e proximidade da saída do mercado de trabalho, geralmente não é mais considerado para medidas de atualização profissional (Walker 1999).

Figura 1: Desenvolvimento da participação das pessoas empregadas por faixa etária (gráfico a partir de dados do IBGE 2001)

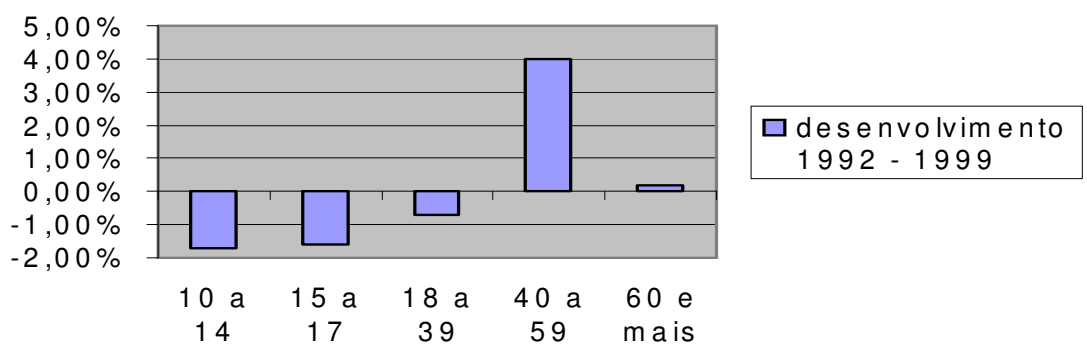

O presente trabalho analisará um conjunto de dados referente ao grupo de trabalhadores mais velhos, confrontando com dados atuais sobre a exclusão digital (Neri, 2003). Com isso será possível conhecer de forma mais diferenciada este grupo específico, suas condições e suas necessidades, bem como as possibilidades de ações de inclusão digital.

\section{Método}

Os dados, que servem de base para este trabalho, foram coletados durante três pesquisas sobre trabalhadores mais velhosi. Durante a primeira pesquisa ${ }^{\text {ii }}$ sobre a imagem do trabalhador mais velhos na indústria metalúrgica foram realizadas 80 entrevistas em 16 empresas. 32 das pessoas entrevistadas pertenciam ao grupo de trabalhadores com mais que 40 anos. As pessoas foram escolhidas por acaso dentro das empresas, atendendo somente a característica de pertencer ao grupo de trabalhadores mais velhos com mais que 40 anos. As outras duas pesquisas ${ }^{\text {iii }}$ foram realizadas durante dois cursos de introdução ao uso do computador para trabalhadores mais velhos, visando estudar os processos de aprendizagem. Durante os cursos foram realizadas entrevistas semiestruturadas, enfocando principalmente experiências escolares e não-escolares de aprendizagem e experiências profissionais. Os participantes destes cursos, seis no primeiro curso e nove no segundo curso, são trabalhadores com mais que 40 anos, que trabalham nas empresas e que participaram na primeira pesquisa.

Para questões de análise, estes dois grupos devem ser tratados de forma diferente. $\mathrm{O}$ primeiro grupo, que foi escolhido por acaso, pode ilustrar características gerais de trabalhadores nesta faixa etária, mesmo não se tratando de um grupo estritamente representativo. O segundo grupo, os participantes dos cursos, pertencem também ao grupo de trabalhadores mais velhos, mas já pela iniciativa de participar de um curso de introdução de computação, os dados deste grupo devem ser analisados de forma especial. 
Entre as múltiplas informações recebidas durante as entrevistas com os dois grupos, foram selecionadas as seguintes categorias para uma análise mais aprofundada, que será enriquecida por informações adicionais obtidas durante as entrevistas:

- idade

- escolaridade

- salário

Em relação à exclusão digital no Brasil, já existem dados e análises mais detalhadas. Sob a coordenação de Neri, um grupo de pesquisadores elaborou um mapa da exclusão digital no Brasil, analisando os dados do IBGE 2000 e do PNAD 2001 (Neri, 2003). Na análise, os dados das nossas pesquisas serão confrontados com estes dados mais gerais sobre a exclusão digital.

\section{Apresentação dos dados e discussão}

\subsection{Idade}

O grupo de trabalhadores mais velhos é uma categoria definida principalmente pela sua idade cronológica. Em relação a "trabalhadores mais velhos", não existe uma idade claramente marcada, que os defina como "mais velhos", mas as pesquisas nesta área utilizam, geralmente a idade de 40 ou 45 anos como limite, a partir do que se fala de trabalhadores mais velhos (veja, por exemplo, Maier 1998). Trata-se de uma idade na qual as pessoas geralmente ainda pertencem à população economicamente ativa, mas onde as perspectivas dos trabalhadores já se aproximam da aposentadoria.

Em relação a nossas pesquisas, encontramos os seguintes dados:

Tabela 1: Idade dos trabalhadores mais velhos

\begin{tabular}{|l|c|c|c|c|}
\hline & idade média & valor mínimo & valor máximo & aposentados \\
\hline grupo 1 $(\mathrm{n}=32)$ & 54,1 anos & 43 anos & 72 anos & $69 \%$ \\
\hline grupo 2 $(\mathrm{n}=15)$ & 46,7 anos & 40 anos & 57 anos & $27 \%$ \\
\hline
\end{tabular}

grupo 1: trabalhadores, entrevistados na pesquisa "Envelhecimento populacional e o mercado de trabalho no Brasil: a imagem do trabalhador mais velho"

grupo 2: trabalhadores que participaram dos cursos "Introdução ao uso do computador"

O que chama a atenção é a abrangência de idade, principalmente no primeiro grupo. Isso demonstra que as pessoas continuam hoje por mais tempo no mercado de trabalho. A permanência no mercado de trabalho não depende do fato de ser aposentado ou não, diferente dos dados em outros países, onde a aposentadoria significa geralmente a saída do mundo de trabalho. Mesmo se existe entre os homens com 60 a 75 anos um declínio forte da participação no mercado de trabalho, impressiona a porcentagem de $33 \%$ de pessoas com 75 anos que continuam trabalhando (60 anos - 62\%). Desta forma fica claro que a participação das pessoas idosas no mercado de trabalho no Brasil está aumentando bastante. Enquanto em 1977, somente 4,5\% da População Economicamente Ativa (PEA) era composta por idosos, este número dublicou para 9\% em 1998 (Camarano 2001).

Em relação à inclusão digital, coloca-se a questão de se trabalhadores nesta faixa etária estariam ainda dispostos a enfrentar cursos para se familiar com o mundo digital. Neste sentido, os dados da primeira pesquisa foram negativos. A atualização profissional é vista, tanto pelos trabalhadores mais velhos, quanto pelos mais jovens como uma 
característica exclusiva de pessoas jovens (Doll, 2002 a). Neste sentido, deve-se contar com dificuldades e resistências para uma inclusão ao mundo digital.

Apesar destes dados, existem outros sinais que apontam para uma maior abertura do que se pensava por parte dos trabalhadores mais velhos. Apesar de todos os preconceitos, que os participantes nos cursos de "Introdução ao uso do computador para trabalhadores mais velhos" mencionaram nas entrevistas, existe um certo interesse em buscar esta atualização. Em uma das empresas, foi necessário mesmo fazer um sorteio para determinar quem poderia participar do curso.

Comparando os dois grupos, fica evidente que, entre os trabalhadores mais velhos, são principalmente os da faixa etária entre 40 e 50 anos os que procuram uma atualização. Para este grupo, a necessidade de se manter no mercado de trabalho para sustentar a família, mas também sonhos profissionais futuros, incentivam-os em procurar conhecimentos considerados necessários no mundo de trabalho atual. As pessoas com mais que 50 anos nos nossos cursos foram pessoas que geralmente já exerciam alguma função de chefia e procuravam conhecimentos específicos para suas funções profissionais atuais.

\subsection{Escolaridade}

A escolaridade é um fator importante em relação ao acesso ao mundo dos computadores, como Neri (2003, p. 37) e seu grupo demonstram em sua pesquisa. As pessoas com até 8 anos de escolaridade possuem raramente acesso ao computador (7,6\% das pessoas com 4 a 8 anos de escolaridade), para pessoas com mais que 8 anos, esta probabilidade aumenta bastante $(17,58 \%)$, e entre as pessoas com mais que 12 anos de escolaridade, mais do que a metade possui acesso ao computador $(58,92 \%)$. Neste sentido,é interessante em nossa pesquisa analisar a escolaridade dos trabalhadores mais velhos.

Tabela 2: Escolaridade dos trabalhadores mais velhos

\begin{tabular}{|l|c|c|c|c|}
\hline & Primário & Ensino Fundamental & Ensino Médio & Ensino Superior \\
\hline grupo 1 $(\mathrm{n}=32)$ & 15 & 4 & 11 & 2 \\
\hline grupo 2 $(\mathrm{n}=15)$ & 2 & 7 & 3 & 3 \\
\hline
\end{tabular}

grupo 1: trabalhadores, entrevistados na pesquisa "Envelhecimento populacional e o

mercado de trabalho no Brasil: a imagem do trabalhador mais velho"

grupo 2: trabalhadores que participaram dos cursos "Introdução ao uso do computador"

Os dados demonstram um amplo leque, de pessoas quase analfabetas a pessoas com formação universitária. A maior parte dos trabalhadores fizeram estudos, que os levaram no máximo até a conclusão do Ensino Fundamental, mas existe também um grupo já considerável que ingressou no Ensino Médio. De fato, estes dados precisam de um detalhamento maior.

Em primeiro lugar, deve-se considerar que a formação escolar está fortemente vinculada ao contexto socio-histórico. Uma comparação entre trabalhadores mais jovens e mais velhos demonstrou que os jovens trabalhadores nas mesmas empresas possuem hoje uma formação escolar maior do que os mais velhos (Doll, 2002 b), refletindo uma maior preocupação com a formação escolar atualmente do que 30 anos atrás. O mercado de trabalho dos anos 70 absorveu, sem maiores problemas, as pessoas mesmo com formação escolar baixa. Isso mudou nos anos 90, principalmente em função da modernização dos processos de produção que exigiram trabalhadores letrados. As 
certificações de qualidade são uma expressão destas mudanças e são a razão pela qual as empresas exigem hoje uma formação mínima do Ensino Fundamental concluído, de preferência Ensino Médio dos seus trabalhadores. Isso significava para os trabalhadores no mercado de trabalho a obrigação de voltar a estudar para se adequar a estas novas exigências ou sair das empresas.

Uma análise das biografias escolares dos trabalhadores do grupo2 demonstra de forma mais detalhada, como as dificuldades com a formação escolar e as pressões atuais de uma formação escolar maior repercutiram nas vidas dos trabalhadores. Para quase todos os trabalhadores mais velhos, a idade escolar foi marcada pelo conflito entre a escola e o trabalho. Isso levou a grande maioria deles a sair da escola ainda durante o Ensino Fundamental, geralmente aliada à necessidade de trabalhar para ajudar à família estavam algumas dificuldades escolares. Na maioria dos casos, a repetição de um ano foi o ponto da evasão escolar. Por outro lado, as novas exigências das empresas nos anos 90 levaram muitos trabalhadores de volta aos estudos, buscando, de várias formas, pelo menos a conclusão do Ensino Fundamental.

Para ações de inclusão digital, estes dados são da maior importância. Principalmente, deve-se considerar que a grande maioria dos trabalhadores não teve uma vida escolar regular, geralmente interrompida e retomada, às vezes, muitos anos mais tarde. Isso significa que os processos de aprendizagem, especialmente em relação a habilidades, como o letramento, provavelmente ficaram comprometidos. Isso significa que atividades que exigem o uso da língua escrita, seja para a leitura, seja para a escrita, podem apresentar consideráveis dificuldades.

\subsection{Salário}

O acesso ao computador não é somente uma questão de vontade de entrar no mundo digital, mas principalmente uma questão de dispor dos recursos necessários para adquirir um computador. Neste sentido, a questão do salário dos trabalhadores é outra questão importante a ser considerada neste contexto.

Tabela 3: Salários dos trabalhadores mais velhos

\begin{tabular}{|l|c|c|c|}
\hline & Média de salário & Salário mais baixo & Salário mais alto \\
\hline grupo 1 $(\mathrm{n}=32)$ & $1.200 \mathrm{R} \$$ & $310 \mathrm{R} \$$ & $2.450 \mathrm{R} \$$ \\
\hline grupo 2 $(\mathrm{n}=15)$ & $1.780 \mathrm{R} \$$ & $300 \mathrm{R} \$$ & $8.000 \mathrm{R} \$$ \\
\hline
\end{tabular}

grupo 1: trabalhadores, entrevistados na pesquisa "Envelhecimento populacional e o mercado de trabalho no Brasil: a imagem do trabalhador mais velho"

grupo 2: trabalhadores que participaram dos cursos "Introdução ao uso do computador"

De novo, encontramos diferenças bastante significativas nos nossos grupos, especialmente no grupo 2. De fato, estes dados precisam algumas explicações. Em relação ao grupo 1, observamos um salário médio relativamente alto, que se explica pelo fato de que dois terços do grupo já eram aposentados, mas continuavam trabalhando. Isto significa que os trabalhadores recebiam, além dos seus salários, ainda a aposentadoria, o que os deixava em uma situação relativamente boa. Em relação ao grupo 2, o número pequeno $(\mathrm{n}=15)$ e o fato de ter uma pessoa com um salário bastante elevado em função do cargo de chefia que ocupa, trazem uma distorção forte para a média. A grande parte dos salários dos participantes variava entre 700 e 1.100 Reais.

Os dados dos trabalhadores demonstram, que a grande maioria ganha um salário razoável, mas com um rendimento em torno de 1.000 reais e sustentando a família, a V. $2 \mathrm{~N}^{\circ} 1$, Março, 2004 
aquisição de um computador não é muito fácil. De fato, em torno de um terço dos participantes, tanto do grupo 1 , quanto do grupo 2 , mencionaram que possuiam um computador em casa. Com isso, o grupo de trabalhadores mais velhos está acima da média do Rio Grande do Sul, pois segundo os dados do PNAD 2001, somente 13,47\% da população deste estado tem acesso ao computador (Brasil: 12,46\%) (Neri, 2003).

Este dado poderia indicar uma maior inclusão digital dos trabalhadores mais velhos do que se pensava inicialmente, porém, nas entrevistas, ficou evidente que o fato de ter um computador em casa não significa automaticamente que a pessoa consiga lidar com o mundo digital. De fato, praticamente todos trabalhadores, que participaram dos cursos, mencionaram que eles tinham adquirido o computador para seus filhos, mas que eles mesmos não sabiam mexer com esta máquina.

Este aspecto é uma característica dos trabalhadores mais velhos. Quando uma pessoa jovem compra um computador, com certeza, ela mesma vai usar. Mas os trabalhadores mais velhos adquirem um computador para seus filhos, o que significa uma oportunidade para estes, porém, não necessariamente a inclusão em si. Este fato demonstra a importância de dois momentos em relação à inclusão digital: por um lado, ter acesso a um computador, por outro lado, ter as competências para usá-lo. Cada aspecto por si só não representa a inclusão digital da pessoa.

\section{Conclusão}

A análise de dados referentes a trabalhadores mais velhos da indústria metalúrgica trouxe alguns aspectos importantes para a inclusão digital deste grupo específico. Em primeiro lugar, contrariando as expectativas e os preconceitos, existe um grupo significativo com forte interesse em ingressar no mundo da informática. Isso significa, que é necessário criar oportunidades para estas pessoas. A atual exclusão digital deste grupos deve-se, provavelmente, à falta de oportunidades de cursos adequados.

Para a realização de cursos deste tipo deve-se considerar, entre outros aspectos, a biografia escolar, geralmente complicada entre os trabalhadores. Isso significa que se deve oferecer um curso, o qual considere as prováveis dificuldades em relação a conhecimentos escolares, especialmente em relação ao uso da língua escrita. Por outro lado, os professores e monitores devem criar um ambiente no curso que possa ajudar a superar possíveis experiências de fracasso anteriormente vivenciadas na escola. Para isso, o trabalho com um sistema de monitores demonstrou ser bastante oportuno, pois facilita a ajuda individual, sem expor o trabalhador na frente do grupo quando ele enfrenta dificuldades. Outro aspecto importante é a consideração que a passagem do mundo análogo para o mundo digital é, para uma pessoa nesta faixa etária, bastante complicada e difícil (Bianchetti, 2001). Para tal precisa-se, por exemplo, bastante espaço para experimentar, repetir e revisar, com um avançar em pequenos passos e levando em consideração o mundo de trabalho, de onde os participantes vêm. Principalmente, mister um ambiente onde se possam superar os medos e as angústias que muitos trabalhadores demonstram em relação à técnica digital (Baracat, Marquié, 1994).

Os dados sobre os salários dos trabalhadores mais velhos demonstraram que, para a grande maioria dos trabalhadores, a aquisição de um computador significa um investimento muito grande frente aos seus salários. Outro resultado interessante, foi que a existência de um computador na casa de um trabalhador mais velho não significa sua inclusão digital, pois, geralmente, o computador é mais usado pelos filhos. Sem cursos adequados, o computador sozinho não garante a inclusão. Em relação a isso, alguns

6 - V. $2 \mathrm{~N}^{\circ} 1$, Março, 2004 
trabalhadores mencionaram experiências interessantes nas suas empresas, onde eles podem, durante os intervalos, ter acesso ao computador. Isso é especialmente importante para trabalhadores, que possuam interesse e talvez até já fizeram cursos, mas que ainda não conseguem adquiri um computador próprio.

Finalmente, não se deve esquecer, no debate sobre a inclusão digital, que além do acesso a um computador e dos conhecimentos sobre o seu manuseio, as pessoas precisam aprender a usar o computador como uma ferramenta para uma atividade, que seja importante para elas, de forma que o computador não seja somente uma diversão sem maior sentido.

\section{Referência bibliográfica}

BARACAT, B.; MARQUIÉ, J.C. Training the middle-aged in new computer technology using signal detection theory in a real-life word-processing learning situation. In: SNEL, Jan (org.). Work and Aging: A European Perspective. London: Taylor \& Francis, 1994, p. 197-211.

BIANCHETTI, Lucídio. Da chave de fenda ao laptop. Tecnologia digital e novas qualificações: desafios à educação. Petrópolis: Vozes, 2001.

CAMARANO, Ana Amélia. O idoso brasileiro no mercado de trabalho. Texto para discussão nº 830. Rio de Janeiro: IPEA, 2001.

DOLL, Johannes. Ältere Arbeitnehmer in Brasilien. In: Karl, Fred; Zank, Susanne (orgs.). Zum Profil der Gerontologie. Kasseler Gerontologische Schriften Band 30. Kassel: Universitätsbibliothek, 2002, 99-105.

DOLL, Johannes. The situation of elderly workers in Brazil. Annual Bulletin of the TALIS Network, $\mathrm{n}^{\mathrm{o}}$ 12, 2002, 3-13 .

FEE. Informe Pesquisa de Emprego e Desemprego. Fundação de Economia e Estatística Siegfried Emanuel Heuser, ano 11, junho 2003.

FRIGOTTO, Gaudêncio. A dupla fase do trabalho: criação e destruição da vida. In: Frigotto, Gaudêncio; Ciavatta, Maria (orgs.). A experiência do trabalho e a educação básica. Rio de Janeiro: DP\&A, 2002, p. 11-27.

IBGE. Brasil em números. Rio de Janeiro, IBGE, 2001.

JORNADA, Maria Isabel Herz; STERNBERG, Sheila S. W.; ZIMMERMANN, Ilaine; ELTZ, Ana S. Impactos sociais e territoriais da reestruturação econômica no Rio Grande do Sul: Impacto das inovações tecnológicas e organizacionais sobre o perfil da mão-de-obra e sobre os requerimentos de qualificação: o caso da indústria mecânica no RS. Porto Alegre: Fundação de Economia e Estatística Siegfried Emanuel Heuser, 1999.

KUENZER, Acácia Zeneida. Educação, linguagens e tecnologias: as mudanças no mundo do trabalho e as relações entre conhecimento e método. In: CANDAU, Vera Maria (org.). Cultura, linguagem e subjetividade no ensinar e aprender. Rio de Janeiro: DP\&A, 2001, p. 135-160. 
LEHR, Ursula. A revolução da longevidade: impacto na sociedade, na família e no indivíduo. Estudos Interdisciplinares sobre o Envelhecimento, Porto Alegre, v. 1, 1999, p. 7-35.

MAIER, Gabriele. Formen des Erlebens der beruflichen Situation: Ein Beitrag zur Innovationsfähigkeit älterer Arbeitnehmer. Zeitschrift für Gerontologie und Geriatrie, 1998, vol. 31, p. 127-137.

MOREIRA, Morvan de Mello. O envelhecimento da população brasileira: intensidade, feminização e dependência. Rev. Bras. Estudos Pop., Brasília, 15 (1), 1998, 79-94.

NERI, Marcelo Côrtes (org.). Mapa da exclusão digital. Rio de Janeiro: FGV/IBRE, CPS, 2003.

WALKER, Alan. Managing an Ageing Workforce. A Guide to Good Practice. Luxembourg: Office for Official Publications of the European Communities, 1999.

\footnotetext{
i O grupo de pesquisa, vinculado à linha de pesquisa "Trabalho, movimentos sociais e educação" do PPGEDU/UFRGS conta atualmente com os seguintes participantes: Johannes Doll (professor PPGEDU, lider do grupo), Caroline Stumpf Buaes (mestranda PPGEDU), Viviane Loeser (mestranda PPGEDU), Anne Carolina Ramos (mestranda PPGEDU), Ceris Angela Paulo (professora FACCAT), Gustavo Caetano de Mattos Mano (bolsista BIC/UFRGS), Cláudia da Silva Porto (bolsista CNPq/UFRGS), Lessara Barbosa de Aguiar (pesquisadora voluntária/UFRGS), Penélope Rocha Pinto (pesquisadora voluntária/UFRGS).

ii "Envelhecimento populacional e o mercado de trabalho no Brasil: A imagem do trabalhador mais velho", pesquisa realizada em 1999 a 2001, que contava com o apoio da PROPESQ/UFRGS através de uma bolsa de iniciação científica.

iii "Envelhecimento, trabalho e educação: Como aprendem os trabalhadores mais velhos?" e "Envelhecimento, trabalho e educação: Aquisição de conhecimentos de informática e seus reflexos na vida profissional e privada de trabalhadores mais velhos". Estas pesquisas iniciaram em 2002 e contam com o apoio da FAPERGS e com bolsas da iniciação científica da PROPESQ/UFRGS e do CNPq.
} 